\title{
EFFECT OF MOBILE PHONE AND BTS RADIATION ON HEART RATE VARIABILITY
}

\author{
B S Saini ${ }^{1}$, Anukul Pandey ${ }^{2}$ \\ ${ }^{I}$ Associate Professor, ${ }^{2}$ M.Tech, Department of ECE, NIT Jalandhar, Punjab, India, \\ sainibss@gmail.com,anukul66@gmail.com
}

\begin{abstract}
The exponential increase in the use of mobile phone in recent years raised problem associated of health risk with electromagnetic field exposure (EFE).The electromagnetic field emitted by mobile phone and base station transceiver (BTS) may causes biological effects as they are designed to operate in $900-1800 \mathrm{MHz}$ with $12.5 \%$ duty cycle. These radiation is non-ionizing type which may have long term biological effect. There are enormous numbers of known and unknown problems due to EFE few known problems are headaches, heaviness in chest, dizziness etc. This paper deals with cardiovascular effect in relation with EFE by analysis on Heart rate variability (HRV) of 19 healthy male volunteers of age group $23 \pm 4.3$ years in three different condition of EFE level. The ECG was recorded for twenty minutes in order to access all the parameters of HRV. As HRV is not only the best representative of autonomic nervous system (ANS) but also used to access the pathological and physiological conditions. The parameters used in the $H R V$ analysis are frequency domain parameters, sample entropy and scaling exponent. The results clearly show that scaling exponent decreases when higher radiation level is experienced. Mobile phone radiation has caused change in HRV parameters and the change varied with radiation level along with the change in few HRV parameter significance as the $p$ values is $\leq 0.05$. The significant change in the scaling exponent of DFA promises there is shift of RR time series towards higher HRV at the elevated radiation exposure.
\end{abstract}

Index Terms: Autonomic nervous system, Effect of wireless network radiation, Electromagnetic field exposure, Heart rate variability, Scaling exponent, Sample entropy, Frequency domain parameter.

\section{INTRODUCTION}

Heart rate variability (HRV) is a non-invasive clinically significant physiological and psychological marker of cardiac and autonomic nervous system (ANS) [1,2]. Linear and nonlinear techniques can be used to analyze $\operatorname{HRV}[3,4]$. Controversial results have been obtained from last few studies. Some are claiming no significant effect of mobile phone on ANS [5], and some studies shows there was change in ANS activity with reference of HRV parameters [6-7]. Electromagnetic fields (EMF) generated from the either mobile phones or base station transceiver (BTS) referred to wireless network radiation in communication, effect were examined on the basis of HRV parameters.

The Mobile Phones (MP) are low power radio devices which work on electromagnetic fields (EMFs), in the frequency range of $900-1800 \mathrm{MHz}$ signal pulsed at $217 \mathrm{~Hz}$ with pulse width of $577 \mu$ s and duty cycle of $12.5 \%$ [3,21-23] . Radiation Power of MP is much higher at the beginning of communication and changes from $2 \mathrm{~W}$ to $100 \mathrm{~mW}$ after the beginning of communication. The output power of MP is at minimal when the MP is at standby mode. The increase in number of electro sensitivity with year in different part of the world is also reported [8-10].
Exposition to high power RF energies may lead to negative thermic effects and cause skin burns, cataracts. With increase in use of MP in day today activities, studying the effect of exposure to EMF has become important. Survey studies have shown that MPs may cause headache, extreme irritation, forgetfulness, blurring of vision, lacrimation , affects brain activity. Some studies suggest electromagnetic fields emitted by cellular phones may affect working of implantable pacemakers [11-14].

Generally MP are held close to head, this might affect Autonomic nervous system (ANS) by close brain heart connection which modulates the cardiac pacemaker and provides beat to beat regulation of cardiovascular rhythm [15]. The aim of present study is to analyze and determine the effect of MP and BTS radiation on ANS using HRV. Since ANS is a non linear system, therefore HRV signal has been analyzed using non linear method Detrended Fluctuation Analysis (DFA) along with Sample Entropy. The study was carried out on nineteen subjects in three different conditions.

\section{DATA ACQUSITION}

In the present study the Electrocardiogram (ECG) of 19 healthy male volunteers in the age group of $23 \pm 4.3$ years, under three different test conditions namely i) 800 meters from 
nearest BTS without mobile phone ii) 50 meters from nearest BTS without mobile phone iii) 800 meters from nearest BTS with mobile phone near chest in calling mode are recorded for 20 min using Zephyr BioharnessTM physiological monitoring system. Additionally, in case iii) the maximum EMF or RFR exposure was observed i.e. power density $1.65 \pm .32 \mathrm{~W} / \mathrm{m} 2$ (watt per meter square) so, this case named as calling mode exposure period. In case ii) the RFR exposure i.e. power density $2.08 \pm .27 \mathrm{~mW} / \mathrm{m} 2$ (milliwatt per meter square) in less than calling mode exposure but greater than case i so case ii) named as moderate exposure. In case i) the minimal RFR exposure i.e. power density $0.49 \pm .12 \mathrm{~mW} / \mathrm{m} 2$ (milliwatt per meter square) was minimal named as least exposure. Volunteers are selected based on having no genetically diseases and any addiction prior to the ECG recording.

In order to make the experiment reliable, the following issues were considered. During recording duration, the volunteers are in easy sitting mode and they are advised to neither make any bodily movement $[16,17]$ nor try to speak anything even in 3rd condition in order to avoid any recording artifacts. The same environmental conditions were observed along with same mobile phone having specific absorption rate (SAR) 1.2 $\mathrm{W} / \mathrm{kg}$ [18],which is within the ICNIRP [19] limit used during recording.

The radiofrequency radiation (RFR) was measured at the testing sites using Radio Frequency Electromagnetic Field (RF EMF) strength meter $(50 \mathrm{MHz}$ to $3.5 \mathrm{GHz})$ in watt per square. However, the cardiovascular effect may depend on the degree of acceptance of RFR exposure by the living body species not on the RFR exist in space [13].

\section{METHOD}

The recorded data set of 19 volunteers in three different conditions were used to evaluate cardiac behavior during RFR exposure by HRV estimation using Detrended Fluctuation Analysis (DFA), Sample Entropy and Power Spectral Density (PSD) computation which is called also called spectral analysis of HRV.

\section{HRV ANALYSIS}

The recorded data set of 19 volunteers in three different conditions were used to evaluate cardiac behavior during RFR exposure by HRV estimation using Detrended Fluctuation Analysis (DFA), sample entropy and power spectral density (PSD) computation which is called also called spectral analysis of HRV.

The RR time series were preprocessed by automatic filters which exclude some samples of original RR time series which differs $20 \%$ from previous samples [1]. As replacing the samples manually may cause undesirable effects [5].

\subsection{Spectral Analysis}

The PSD analysis deals with periodic oscillation of heart rates for different frequencies and amplitudes relative power in heart's sinus rhythm [20]. The PSD can be computed using parametric and non-parametric methods. Both methods provide comparable results while non-parametric analysis uses autoregressive estimation gives smoother easy calculation of spectral components but needs to verify the suitability and complexity, in contrast the non-parametric method uses simple algorithm Fast fourier Transform (FFT) having high computation speed and easy in implementation. In this paper we use the non-parametric based estimation of spectral components [21]. The result of FFT of RR times series is complex number its squared modulus gives the spectral power. The PSD consist of frequency band $0-.5 \mathrm{~Hz}$ as this study deals with short term recording the spectral components can be visualizes are very low frequency (VLF), low frequency (LF), high frequency (HF). The spectral components are evaluated in range of frequency but the amplitude is the area under that frequency band called power spectral density as represented in table 1. The unit of spectral components are squared millisecond (ms2) or can be represented by normalized unit (n.u) according to following normalizing equation 1 [1].

$$
L F \text { or } H F(n . u)=\frac{L F \text { or } H F\left(m s^{2}\right)}{\text { total power }-\operatorname{VLF}\left(m s^{2}\right)} \times 100
$$

Table-1 spectral components of HRV

\begin{tabular}{|l|l|l|l|}
\hline Variable & Unit & Description & $\begin{array}{l}\text { Frequency } \\
\text { range }\end{array}$ \\
\hline $\begin{array}{l}\text { Total } \\
\text { power }\end{array}$ & $\mathrm{ms} 2$ & $\begin{array}{l}\text { Variance of all RR } \\
\text { intervals }\end{array}$ & $\begin{array}{l}\text { approximatel } \\
\mathrm{y}<0 \cdot 4 \mathrm{~Hz}\end{array}$ \\
\hline VLF & $\mathrm{ms} 2$ & $\begin{array}{l}\text { Power in the very low } \\
\text { frequency range }\end{array}$ & $<=0.04 \mathrm{~Hz}$ \\
\hline LF & $\mathrm{ms} 2$ & $\begin{array}{l}\text { Power in the low } \\
\text { frequency range }\end{array}$ & $0.04-0.15 \mathrm{~Hz}$ \\
\hline HF & $\mathrm{ms} 2$ & $\begin{array}{l}\text { Power in the high } \\
\text { frequency range }\end{array}$ & $0.15-0.4 \mathrm{~Hz}$ \\
\hline
\end{tabular}

LF consist of both sympathetic along with parasympathetic activity behavior. While HF give the measure of efferent nature of vagal activity [2]. HF having information about parasympathetic activity. LF/HF ratio was also calculated which provides the information about the symptho-vagal balance.

\subsection{Deterend Fluctuation Analysis (DFA)}

As the RR time series is non-stationary signal non linear analysis is recommended for better results DFA is one of nonlinear method for HRV, so the DFA calculates root mean square fluctuation of detrended time series [22]. DFA is used to simulating for various biological and physiologic time series and it also permits to calculate the self-similarity of RR- 
time series [23]. In the method of DFA the RR time series is integrated as equation 2 and then vertical characteristic scale was measured.

$$
y(k)=\sum_{i=1}^{k}\left[R R(i)-R R_{\text {avg }}\right]
$$

For measurement of vertical characteristics the time series was divided in boxes of equal length $\mathrm{n}$ which represents the trend in that box and local trend are removed by subtraction of yavg.(average value of integrated time series in that box) [24]. In each box a least square fit to data is used to represent the trend, $\mathrm{yn}(\mathrm{k})$ represent the straight line in particular box. The root mean square of integrated fluctuation and detrended RR times series is given by following equation, which will be calculated for every window [22].

$$
F(n)=\sqrt{\frac{1}{N} \sum_{k=1}^{N}\left[y(k)-y_{n}(k)\right]^{2}}
$$

$\mathrm{N}$ is the overall length of RR series and $\mathrm{F}(\mathrm{n})$ will increase with the box size. The slope of line relating $\log (\mathrm{F}(\mathrm{n}))$ to $\log (\mathrm{n})$ give the value of scaling exponent $(\alpha)$. An $\alpha$ of 0.5 corresponds to white noise, $\alpha=1$ represents $1 / \mathrm{f}$ noise and $\alpha=1.5$ indicates Brownian noise or random walk. Scaling exponent having potency for diagnostic and prognostic abilities with various type of cardiac diseases.DFA method gives superior results with respect to spectral analysis for analysis of HRV in patient with sleep apea [13].

\subsection{Sample Entropy (Sampen)}

Entropy basically deals with randomness, when applied to time series it calculates the dynamics of time series. Approximate entropy (ApEn) was introduced and calculated for regularity measurement for same predefined time window and compare other group for same time length [28]. ApEn, for regular series is expected to having low value while complex signal having higher ApEn values [29]. As ApEn's having self its dependence on the data size make its application limited and alternative, which more robust amenable to short data size sample entropy (SampEn) was introduced by Richmann and Moorman [26,29].

Time series complexity can be measured using sample entropy with less computation time as data set is low and applied to physiological signal. The negative natural logarithm of the conditional probability that two sequences similar for $\mathrm{m}$ points at the next point with a tolerance $r$, where self-matches are excluded [27] was calculated for time series. For sample entropy analysis, the parameter $(\mathrm{m}, \mathrm{r})$ were set according to literature[25]. Where, $\mathrm{N}$ is the length of the time series. In this, we computed SampEn by the values of 2 for $m$ and 0.2 for $r$.

\section{RESULT AND DISCUSSION}

Maximum, mean and minimum instantaneous heart rate did not change significantly in either of least exposure to maximum exposure the values are tabulated in table 2 , which confirms the previous study [6,25]. The paired student-t test was carried out to evaluate the statistical significance. The results obtained using DFA which is a nonlinear HRV gives the statistically significant result $\mathrm{p}$-value $(\mathrm{p}<.05)$ in contrast to the reference [25] because of they considered as the mobile phone is in standby mode in this paper the experimentation was carried out in thousand times higher level RFR exposure exposure which is the general case i.e. mobile phone in calling mode.

Table 2: Instantaneous heart rate in three conditions

\begin{tabular}{|l|l|l|l|l|}
\hline Heart rate & $\begin{array}{l}\text { Least } \\
\text { exposure }\end{array}$ & $\begin{array}{l}\text { Moderate } \\
\text { exposure }\end{array}$ & $\begin{array}{l}\text { Calling } \\
\text { mode } \\
\text { exposure }\end{array}$ & $\begin{array}{l}\text { p- } \\
\text { value }\end{array}$ \\
\hline Mean & $80.67 \pm 8.1$ & $81.29 \pm 7.9$ & $80.99 \pm 8.1$ & ns \\
\hline Maximum & $94.78 \pm 7.2$ & $97.16 \pm 10.4$ & $93 \pm 6.5$ & ns \\
\hline Minimum & $71.26 \pm 8.7$ & $73.11 \pm 11.1$ & $72.42 \pm 7.8$ & ns \\
\hline
\end{tabular}

$\mathrm{ns}=$ not significant

When the RFR exposure is moderate the scaling exponent $(\alpha)$ is showing the same behaviour as in Ahamed et al.'s study being statistically insignificant [25].

The mean value of scaling exponent $(\alpha)$ in table 2 is low in case of least exposure case, highest in the case of maximum RFR exposure and low in moderate case as well. The above study indicates that $\alpha$ value of HRV increases with statistical significance, when mobile phone sited near chest in calling mode with respect to least radiation and also $\alpha$ value of HRV is not significant if the living got exposed by wireless transmitter station, BTS was considered in this paper. This can be well justified by statistical analysis as p-value is $\leq_{0.05 \text { for }}$ calling mode exposure condition.

Table 3: HRV parameters in three conditions

\begin{tabular}{|l|l|l|l|l|l|}
\hline $\begin{array}{l}\text { paramet } \\
\text { ers }\end{array}$ & $\begin{array}{l}\text { Least } \\
\text { exposure }\end{array}$ & $\begin{array}{l}\text { Moderate } \\
\text { exposure }\end{array}$ & $\begin{array}{l}\text { Calling } \\
\text { mode } \\
\text { exposure }\end{array}$ & $\begin{array}{l}\text { p- } \\
\text { val1 }\end{array}$ & $\begin{array}{l}\text { p- } \\
\text { val2 }\end{array}$ \\
\hline LF (n.u) & $\begin{array}{l}43.99 \pm \\
16.79\end{array}$ & $\begin{array}{l}46.56 \pm \\
15.79\end{array}$ & $\begin{array}{l}45.19 \pm \\
18.3\end{array}$ & 0.21 & 0.39 \\
\hline HF (n.u) & $\begin{array}{l}56.01 \pm \\
16.79\end{array}$ & $\begin{array}{l}53.44 \pm \\
15.79\end{array}$ & $\begin{array}{l}54.81 \pm \\
18.4\end{array}$ & 0.20 & 0.38 \\
\hline LF/HF & $0.96 \pm .64$ & $1.09 \pm .41$ & $1.05 \pm .74$ & 0.24 & 0.31 \\
\hline $\begin{array}{l}\text { Sample } \\
\text { entropy }\end{array}$ & $1.66 \pm .24$ & $1.58 \pm .40$ & $1.68 \pm .25$ & 0.21 & 0.42 \\
\hline$\alpha$ & $0.91 \pm .09$ & $0.92 \pm .11$ & $0.96 \pm .09$ & 0.53 & 0.02 \\
\hline
\end{tabular}


$\mathrm{n} . \mathrm{u}=$ normalised unit, $\alpha=$ scaling exponent in DFA, $\mathrm{p}$-val1 = statistical comparison between least and moderate exposure, p-val2 = statistical comparison between least and calling mode exposure

The mean value of sample entropy in table 2 was low in 11 subjects in case of maximum RFR exposure and was high in 9 subjects when it was compared with the least RFR exposure. The statistical significance sample entropy value was not made because of higher $\mathrm{p}$-value.

The mean value of LF component increases in normalized unit indicated in table 2 which shows that with exposure RFR of either moderate or maximum exposure which is calling mode exposure in this paper also increase in parasympathetic and sympathetic tone. LF is marker of both parasympathetic and sympathetic activity [1-3]. But the change in LF component is selective in 8 volunteers it increases in calling mode case were as in 13 volunteers it decreases in moderate exposure case.

The mean value of HF component decreases in normalized unit indicated in table 2 which shows that with exposure RFR decrease in parasympathetic behaviour of ANS.

The HF is marker of efferent vagal activity [1-4]. But the change in HF component is not stabilized in all the volunteers as it decreases in 11 volunteers during calling mode RFR exposure where as it decreases in 13 volunteers during moderate RFR exposure mode.

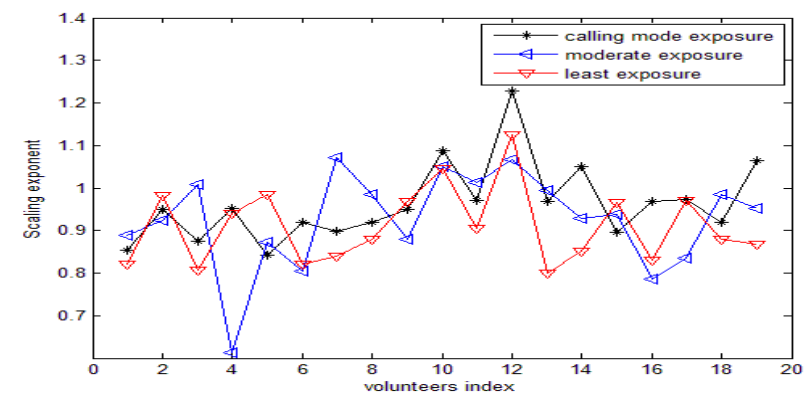

Figure 1: Change in the Scaling exponent.

Overall, HF decreases and LF increases indicating that there is reduction in vagal activity and increase in sympathetic behaviour of ANS. Obviously there will be increase in mean value of $\mathrm{LF} / \mathrm{HF}$ ratio which can be interpreted as the sympathetic modulation increases with respect to parasympathetic activity. But statistical significance was not observed in either of exposure case.

It can be seen that scaling exponent show significant change during high RFR exposure as mobile phone emit highest power during calling mode, rest in other conditions the low RFR exposure does not having significant impact on HRV parameters.

\section{CONCLUSIONS}

The potency of RFR exposure by wireless network on cardiac behaviour based on non linear HRV scaling exponent was examined. The parameters namely, DFA scaling exponent $\alpha$ increases with $\mathrm{p}$-value $<0.05$ when maximum RFR exposure was recorded, otherwise at low level RFR exposure the $\alpha$ value loses its statistical significance.

This study was limited to the volunteers in least exposure considered as control group. Which mean no additional control group comparison was made. In order to come up with more detailed cardiological effect of wireless network radition more experimentation should be conducted on more number of subject along with more number different of RFR exposure levels..

\section{REFERENCES}

[1] Task Force of the European Society of Cardiology and the North American Society of Pacing and Electrophysiology, Heart rate variability - Standards of measurement, physiological interpretation, and clinical use, European Heart Journal, 1996, vol. 17, pp. 354381.

[2] Juan Sztajzel "Heart rate variability: a noninvasive electrocardiographic method to measure the autonomic nervous system,"SWISS MED WKLY vol.134, pp 514 - 522, 2004.

[3] Singh D., B.S. Saini "Heart Rate Variability-A Bibliographical Review", IETE vol.54 no.3, pp209216.

[4] U. Rajendra Acharya, K. Paul Joseph,N. Kannathal, Choo Min Lim, Jasjit S. Suri, "Heart rate variability: a review," Med Bio Eng Comput vol.44, 1031-1051, 2006.

[5] Oliver Faust ,U. Rajendra Acharya, "Effects of Mobile phone radiation on cardic health," world scientific

[6] D. Yilmaz and M. Yildiz "Analysis of the Mobile Phone Effect on the Heart Rate Variability by Using the Largest Lyapunov Exponent," J Med Syst 34:10971103.2010.

[7] R. Andrzejak, R. Poreba, M. Poreba, A. Derkacz, R. Skalik, P. Gac, B. Beck, A. Steinmetz-beck and W. Pilecki, "The influence of the call with a mobile phone on heart rate variability parameters in healthy volunteers." Industrial Health 2008 vol.46,pp.409-417.

[8] N.K. Panda,J.R. Bakshi,J.Bakshi,S. Munjal, "Audiologic disturbances in long-term mobile phone users,"J Otolaryngol Head Neck Surg. 2010 Feb;39(1):5-11.

[9] "Letter to the Editor: Will We All Become Electrosensitive?" Electromagnetic Biology and Medicine, 25: 189-191, 2006.

[10] W. Ross Adey, "Biological Effects of Electromagnetic Fields," Journal of Cellular Biochemistry 51:410-416, 1993. 
[11] B. B. Levitt and Henry Lai, "Biological effects from exposure to electromagnetic radiation emitted by cell tower base stations and other antenna arrays," NRC Research Press Environ. Rev. 18: 369-395 (2010)

[12] Biological Effects of Radiation USNRC Technical Training Center.

[13] The INTERPHONE Study Group, "Brain tumour risk in relation to mobile telephone use: results of the INTERPHONE international case-control study," International Journal of Epidemiology 39(3):675-694, 2010.

[14] Report on possible impacts of communication towers on wildlife including birds and bees, Ministry of Environment and Forests, Government of India, 12 October 2011.

http://www.ee.iitb.ac.in/ mwave/workshop.htm

[15] R. U. Achary, C.M. Lim, P. Joseph "Heart rate variability analysis using correlation dimension and detrended fluctuation analysis," ITBM-RBM 23 : 333339. 2002.

[16] "Recording a standard 12-lead electrocardiogram An approved methodology," The society for Cardiological science and technology (2013) online

[17] Luciano Bernardi, MD, Joanna Wdowczyk-Szulc, MD, Cinzia Valenti, MD, Stefano Castoldi, BS, Claudio Passino, MD, Giammario Spadacini, MD, Peter Sleight, MD, "Effects of Controlled Breathing, Mental Activity and Mental Stress With or Without Verbalization on Heart Rate Variability" FRCP Journal of the American College of Cardiology Vol. 35, No. 6, Published by Elsevier Science Inc., 2000.

[18] IEEE Recommended Practice for Measurements and Computations of Radio Frequency Electromagnetic Fields With Respect to Human Exposure to Such Fields, 100 kHz-300 GHZ R2008

[19] ICNIRP guidelines on limits of exposure to static magnetic fields published in: health physics 96(4): 504-514, 2009

[20] M. Malik, T. Cripps, T. Farrell, A.J. Camm. "Prognostic value of heart rate variability after myocardial infarction - a comparison of different data processing methods" Med Biol Eng Comput 1989, vol. 27: 603-11.

[21] Hung-Wen Chiu, "Frequency-domain Heart Rate Variability Analysis Performed by Digital Filters Tsung-Chieh Lee1," Computing in Cardiology vol.37,589-592,2010.

[22] C. Heneghan and G. McDarby, "Establishing the relation between detrended fluctuation analysis and power spectral density analysis for stochastic processes," Phys Rev E. 62(5): 6103-6110, (2000).

[23] V. E. Papaioannou, I. G. Chouvarda, N. K. Maglaveras, and I. A. Pneumatikos, "Study of multiparameter respiratory pattern complexity in surgical critically ill patients during weaning trials," Published online 2011 January 21.
[24] http://en.wikipedia.org/wiki/Detrended_fluctuation_ana lysis

[25] V.I. T. Ahamed, N.G. Karthick, P. K. Joseph, "Effect of mobile phone radiation on heart rate variability," Computer in Biology and Medicine vol.38, pp. 709$712,2008$.

[26] J. S. Richman and J. R. Moorman, "Physiological timeseries analysis using approximate entropy and sample entropy," Am J Physiol Heart Circ Physiol 278: H2039-H2049, 2000.

[27] http://physionet.incor.usp.br/physiotools/sampen/

[28] S.M. Pincus, "Approximate entropy as a measure of system complexity," Proc. Natl. Acad. Sci. USA 88 (6) 2297-2301,( 1991).

[29] D.E.Lake, J.S. Richman, M.P. Griffin, J.R. Moorman, "Sample entropy analysis of neonatal heart rate variability." Am J Physiol Regul Comp Physiol. 283:789-797,2002.

\section{BIOGRAPHIES:}

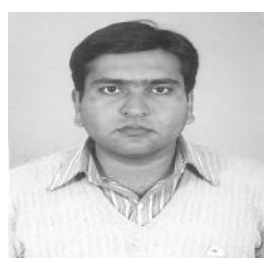

Barjinder S. Saini was born in Jalandhar, India, in 1970. He received his B.Tech and M.Tech. degrees in Electronics \& Communication Engineering in 1994 and 1996 respectively. He then obtained his $\mathrm{PhD}$ degree in Engineering on "Signal Processing of Heart Rate Variability" in

2009 from Dr. B. R. Ambedkar National Institute of Technology, Jalandhar. He is working as Associate Professor in Electronics \& Communication Engineering Department at NIT Jalandhar since 1997. He supervised more than 20 M.Tech dissertations and presently guiding $06 \mathrm{Ph}$. D research scholars. He has published more than 20 research papers in internationally reputed Journals and Conference proceedings. $\mathrm{He}$ has accomplished research projects from government departments. He has also conducted several courses, workshops and international conferences for the benefit of faculty and field engineers. His areas of interest are Medical Image Processing, Digital Signal Processing and Telemedicine and embedded systems. Dr. Saini holds membership of many professional bodies. He is a life member of the IETE.

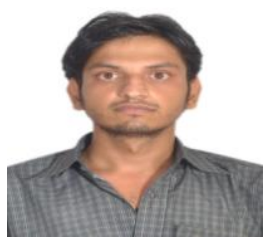

Anukul Pandey has completed B.Tech in Electronics and communication from BPUT Orissa in 2011 and currently pursuing M.tech from National Institute of Technology Jalandhar. 